\title{
Reactive Transport Modeling of Remediation Strategies for Coal Combustion Residuals Sites
}

\author{
PJ NOLAN $^{1}$ AND RENS VERBURG ${ }^{2}$
}

${ }^{1}$ Golder Associates, 18300 NE Union Hill Road, Suite 200, Redmond, WA 98052; pj_nolan@golder.com

${ }^{2}$ Golder Associates, 18300 NE Union Hill Road, Suite 200, Redmond, WA 98052; rens_verburg@golder.com

The identification of remedial alternatives requires a balanced evaluation of cost, sustainability, potential for success, reputational risk, regulatory and other stakeholder acceptance, and expediency. A favored approach among stakeholders for remediation of Coal Combustion Residuals (CCR) is full removal. In many cases, this represents arguably the least sustainable approach to CCR remediation in terms of energy demand, resource allocation, and expedience. This approach may also lead to increased contamination of groundwater due to chemical alteration and physical agitation. However, the long-term consequences of CCR removal are typically not well understood.

In this presentation, we demonstrate a streamlined approach to integrating hydrogeologic and geochemical modeling and data to evaluate CCR remediation strategies. Model development was guided by detailed groundwater, soil, and source characterization, including sequential extraction and mineralogical analysis. The remediation options modeled include: natural attenuation, semi-permeable caps, barrier wall emplacement, geochemical manipulation, and source removal. Results demonstrate the long-term potential for success of each corrective measure, including the consequences of CCR removal.

Notably, the development of a model to realistically characterize source removal proved to be challenging. Geochemical models were developed in a sequential nature in tandem with groundwater models due to a changing physical domain, changing groundwater flow field, and pressure head across the domain. Geochemical parameters were transferred from one model to the next in a near-coupled approach. In addition, a novel approach was applied for incorporating clean attenuating media into the geochemical model to match expected real-world conditions.

This approach demonstrates how integrated modeling techniques can be used to leverage expertise in groundwater flow and geochemical modeling at CCR sites. Remedial performance can be evaluated in a manner that accounts for contaminant attenuation as well as potential adverse consequences. 